\title{
Relación Entre Escoliosis, Sexo y Lateralidad Manual en una Muestra de Escolares
}

\author{
Relationship between Scoliosis, Sex and Handedness in a Sample of Schoolchildren
}

Fernández, M."; Fernández, R.*; Zurita, F.*; Jiménez, C. ${ }^{* * * *}$; Almagià, A..****; Yuing, T.**,***** \& Curilem, C.****

FERNÁNDEZ, M.; FERNÁNDEZ, R.; ZURITA, F.; JIMÉNEZ, C.; ALMAGIÀ, A.; YUING, T. \& CURILEM, C. Relación entre escoliosis, sexo y lateralidad manual en una muestra de escolares. Int. J. Morphol., 33(1):24-30, 2015.

RESUMEN: De todo el conjunto de problemas que se desarrollan en la infancia, no cabe duda que la escoliosis ocupa un puesto importante debido a sus repercusiones en la edad temprana y adulta. El objetivo del estudio fue evaluar la relación existente entre el sexo, escoliosis y lateralidad manual. La muestra estuvo formada por 2822 participantes, con edad comprendida entre 6 y 12 años, pertenecientes a la provincia de Almería. Se utilizaron dos instrumentos de medida, es decir, inventario de lateralidad manual de Edinburgh y la prueba de Adams, para analizar las variables seleccionadas. No se encontraron diferencias estadísticamente significativas entre las variables lateralidad y sexo $(\mathrm{p}=0,106)$ ni entre lateralidad y escoliosis $(\mathrm{p}=0,673)$; pero sí entre escoliosis y sexo $(\mathrm{p}<0,001)$. Como principales conclusiones debemos señalar la importancia en el desarrollo de adecuados programas de intervención teniendo en cuenta el número obtenido de niños con escoliosis, sobre todo en niñas.

PALABRAS CLAVE: Prevalencia; Escoliosis; Obesidad; Escolares; Columna Vertebral.

\section{INTRODUCCIÓN}

De todo el conjunto de problemas que se desarrollan en la infancia, no cabe duda que uno de ellos, la escoliosis, es fuente de preocupación en aquellos padres que comienzan a observar con el transcurso del tiempo como sus hijos pueden terminar desarrollando este tipo de patología, asociada en ocasiones a sintomatología dolorosa (Sato et al., 2011). Dentro del gran número de clasificaciones de escoliosis que podemos encontrar en la literatura científica, la Sociedad para el Estudio de las Enfermedades del Raquis la define como una desviación lateral del raquis con manifestaciones clínicas visibles relacionadas con desplazamiento lateral de la columna vertebral, asociada a la rotación de las vértebras implicadas (Zurita Ortega et al., 2008a). Otra definición interesante es la desarrollada por la Scoliosis Research Society, la cual establece hasta un total de trece variedades distintas de escoliosis morfológicas.

Debemos reseñar que la escoliosis idiopática es la más frecuente de todas. Se trata de un síndrome complejo del desarrollo (Gorman et al., 2012) que aparece en la infancia y produce una inclinación lateral en el plano frontal junto a una rotación vertebral. A pesar de no tener una causa claramente definida, parece tener una causa multifactorial (Pérez-Caballero Macarrón et al., 2006) donde pueden estar implicados el sexo y la edad, así como factores de tipo genético (Grauers et al., 2012).

Respecto a la relación existente entre escoliosis y sexo, de forma general parece evidenciarse una tendencia más alta en hombres a presentar mayor número de escoliosis (Wang et al., 2012), sin embargo también se debe tener en cuenta que en ocasiones, los resultados obtenidos a este respecto no han sido estadísticamente significativos (Zurita Ortega et al., 2008b). Respecto a esta temática, otros estudios han observado que tras la maduración ósea se da una mayor progresión escoliótica en varones (Miller et al., 1984).

Se puede establecer una clasificación de la escoliosis idiopática atendiendo a varios parámetros, es decir, el número y patrón de las curvas, su magnitud y la edad de presentación (Álvarez García de Quesada \& Nuñez Giralda, 2011). En lo que respecta a su clínica, se entiende la necesidad de un diagnóstico precoz y por tanto la inspección periódica en las escuelas para detectar esta deformidad (Zurita

\footnotetext{
" Laboratorio Funcional, Facultad de Ciencias, Enfermería y Fisioterapia, Universidad de Almería, Almería, España.

** Laboratorio de Didáctica Corporal, Departamento de Función Corporal, Facultad de Ciencias de la Educación, Universidad de Granada, Granada, España.

*** Laboratorio de Anatomía Humana, Escuela de Kinesiología, Facultad de Ciencias de la Salud, Universidad Santo Tomás, Viña del Mar, Chile.

${ }^{* * * * *}$ Laboratorio de Antropología Física y Anatomía Humana, Instituto de Biología, Facultad de Ciencias, Pontificia Universidad Católica de Valparaíso, Valparaíso, Chile.
} 
Ortega et al., 2008b) con la finalidad de disminuir una posible intervención quirúrgica (Bunge et al., 2008). Siguiendo con lo expuesto, el principal aspecto clínico de la escoliosis es la deformidad del tronco, que se determina desde dos puntos de vista: dorsal y ventral (Kapandji, 2007).

Existen distintas técnicas para dictaminar la presencia o no de escoliosis, resaltamos entre otras la entrevista personal, que recoge información relativa a la deformidad vertebral, la historia familiar, la exploración física, el uso de test y pruebas indirectas de detección, etc. También resulta de importancia realizar medidas torácicas y aplicar un adecuado examen clínico y radiológico (Yagi et al., 2012; Watanuki et al., 2012).

De todos los problemas o patologías presentes en la población infantil, no cabe duda que los problemas de espalda son comunes y preocupan en gran medida tanto a padres como instituciones públicas y privadas, debido principalmente a las consecuencias psicológicas y físicas que están lesiones implican y conllevan (Bull \& Grogan, 2010). Concretamente, la escoliosis es un tipo de patología vertebral que puede favorecer por ejemplo en población infantil, una disminución en la calidad de vida (Jalanko et al., 2011) y dolor (Pärtan et al., 2010).

Se debe reparar que el concepto de lateralidad está muy ligado a la dominancia hemisférica (Fonseca-Pedrero et al., 2007). Desde un punto de vista motor, como predomino funcional, se entiende la preferencia para utilizar una de las partes simétricas del cuerpo (Rigal, 1988). También podemos conceptuar la lateralidad como la asimetría en el grado de desarrollo fisiológico de los hemisferios cerebrales izquierdo o derecho en cada clase de actividades, principalmente en el lenguaje y en las funciones simbólicas (Tichy \& Belacek, 2009).

Es importante reseñar que la lateralidad no es un proceso estático y fijado estructuralmente, sino que se halla en equilibrio fisiológico y dinámico. Así, el campo de la Psicología se centra en el estudio del origen de la lateralidad, teniendo en cuenta los factores biopsicosociales y la importancia de la intervención educativa (Del Valle Díaz \& De la Vega Marcos, 2007).

No cabe duda que el proceso de lateralidad es madurativo y desde el nacimiento existen reflejos que pueden indicar la lateralidad del niño. Los primeros indicios de preferencia lateral aparecen entre los 12 meses y los 5 años, siendo uno de los primeros hábitos de los niños el chuparse el dedo, manipular objetos e imitar. A los 2 años, la mayoría de los niños tienen preferencia en el uso de la mano derecha (Terrones González et al., 2003), estableciéndose una clara diferencia durante los 3 años siguientes, de modo que la dextralización en niños diestros es bastante clara a los 5 años (Mcmanus, 2007) y a los 6 años, la mayoría de los niños han desarrollado una clara preferencia (Healey, 2006).

El dominio del habla, y probablemente también de otras facultades, se establece firmemente en uno de los hemisferios hacia los 10 años de edad y no puede transmitirse al otro posteriormente. La edad a la que tiende a ser más clara la lateralización es entre los 9 y 11años, culminando a los 12 años, aunque a partir de los 8 años el niño zurdo está definido (Monge Crespo, 2000). Por otro lado, investigaciones sobre la preferencia podal han dado como resultado que en tareas sencillas, el 50\% de los niños utilizan el miembro inferior contralateral; sin embargo en tareas más complejas la preferencia podal se haya acorde con la dominancia manual (Tichy \& Belacek).

Respecto a los factores que influyen en el desarrollo de la lateralidad se establecen fundamentalmente 3 posturas diferenciadas. En primer lugar, las teorías hereditarias consideran que la preferencia lateral es exclusivamente de origen neurológico (Ferre et al., 2006). En segundo lugar las teorías ambientalistas le dan mayor importancia a las influencias del entorno familiar, escolar y social (Springer \& Deutsch, 2006). Finalmente están las teorías mixtas, que integran ambas posturas, dando importancia tanto a los factores genéticos como ambientales (Castañer \& Camerino, 1993).

Teniendo en cuenta el escaso número de investigaciones encontradas en la literatura que establecen una posible relación entre escoliosis, sexo y lateralidad hemos planteado un estudio cuyo objetivo principal es establecer la prevalencia de escoliosis en 2822 escolares de la provincia de Almería y su grado de relación con el sexo y lateralidad manual.

\section{SUJETOS Y MÉTODO}

Se contó con un total de 2822 participantes con una media de edad de 8,35 años (rango entre 6 y 12 años) pertenecientes a la provincia de Almería. Se realizaron además análisis correlacionales para analizar el grado de dependencia entre las distintas variables objeto de estudio, utilizándose para ello el programa estadístico SPSS 18.0.

Para la realización del estudio se tuvieron en cuenta las siguientes variables descriptivas: i) Sexo, Masculino y femenino, ii) Escoliosis, Dividido en dos categorías: ausencia o presencia, y iv) Lateralidad manual. Se establecieron en este caso dos categorías: diestro y zurdo. 
Las variables del presente estudio fueron registradas mediante dos instrumentos de valoración:

1) Prueba de Adams. Considerado uno de los recursos más utilizados en la detección de las alteraciones ortopédicas del raquis (Zurita Ortega et al., 2008a). El procedimiento relacionado con este test consiste en colocar al participante en posición de bipedestación, con el torso desnudo, descalzo, las rodillas extendidas y los pies juntos. Después se pide al individuo realizar una flexión anterior de tronco de $50^{\circ}$ a $65^{\circ}$ según se explore la giba dorsal o protuberancia lumbar respectivamente. Los brazos se posicionan en suspensión vertical con las palmas de ambas manos en oposición. El explorador se sitúa en sedestación en el plano frontal anterior o posterior respecto del sujeto a explorar, detectando la giba y/o protuberancia mediante visión tangencial del contorno. El aumento del relieve dorsal o lumbar genera una imagen de la giba o protuberancia que en el caso de la escoliosis será asimétrica, este incremento se considera signo positivo que indica en este caso presencia de escoliosis.

2) Inventario de Lateralidad Manual de Edinburgh (Oldfield, 1971). Ampliamente utilizado en investigación Evalúa la preferencia manual a través de 10 actividades y clasifica a los participantes según un cociente de lateralidad (CL). En nuestro estudio solamente se han considerado dos niveles (diestro o zurdos), enclavando en esta última categoría aquellos individuos que eran bilaterales o ambidiestros.

Previa aprobación del estudio por parte de un Comité Ético Institucional y teniendo en cuenta las normas de Helsinki de 1975, se dispuso finalmente con un total de 19 centros escolares pertenecientes a la provincia de Almería para la obtención de la muestra. Se intentó en todo momento conseguir una muestra representativa y homogénea, para ello se estudiaron las características de cada zona y se seleccionaron los Centros de Educación de las diferentes áreas establecidas, en función de sus características (líneas, tipo de centro y características del alumnado).

El siguiente paso consistió en entregar una carta-solicitud a cada uno de los centros educativos, explicando todo el procedimiento a seguir. Se solicitó la colaboración del colegio y la aceptación de todos los miembros implicados (consejo escolar, profesores, padres, servicios médicos, etc.). Se concertó una entrevista personal con los directores de los distintos centros de Enseñanza Primaria seleccionados y con los servicios médicos correspondientes a cada área, informándoles del trabajo de investigación que se pretendía llevar a cabo

Una vez aceptada la propuesta y firmado el consentimiento informado por cada uno de los padres, se acordó con el centro el lugar a utilizar (gimnasio o aula multiuso con habitación adjunta) y las normas a respetar (principalmente atuendo deportivo) para la realización de la prueba, la cual consistía en la aplicación individual tal y como se indica en el apartado instrumentos, del prueba de Adams (tiempo estimado de 3 minutos) en primer lugar y a continuación el Inventario de Lateralidad (tiempo estimado de 3 minutos). Las variables fueron registradas sólo por un investigador. Respecto a la temporalidad, la fecha de los registros tuvo lugar entre Enero y Marzo del 2012.

La selección definitiva de la muestra se realizó por muestreo consecutivo, atendiendo a la composición natural de los grupos, tomando como referencia un criterio de inclusión, es decir, estar matriculado en Educación Primaria. En todos los casos y para mantener el anonimato, la identificación de los participantes se realizó mediante codificación numérica en una ficha de registro personal.

Para analizar la hipótesis de normalidad y homogeneidad de la varianza se utilizaron las pruebas de Kolmogorov Smirnov y Levene. Se realizó un análisis descriptivo de frecuencias teniendo en cuenta las medias y porcentajes. También se hicieron análisis descriptivos mediante tablas de contingencia utilizando en este caso el estadístico chi-cuadrado de Pearson. El programa estadístico utilizado fue el SPSS 18.0.

\section{RESULTADOS}

Respecto a los análisis descriptivos de los 2822 alumnos analizados, un 51,8\% ( $\mathrm{n}=1463)$ pertenecían al sexo masculino y un $48,2 \%(n=1359)$ al sexo femenino. Presentaban escoliosis un 36,3\% de la población ( $n=1023)$, mientras que un $63,7 \%(n=1799)$ no tenían esta patología. Siguiendo con lo expuesto, un $29 \%(\mathrm{n}=435)$ del total de participantes del sexo masculino presentaban escoliosis y en el caso del sexo femenino un $44 \%(\mathrm{n}=598)$. En lo referente a la variable lateralidad un 90,8\% $(n=2562)$ eran diestros y $9,2 \%(n=260)$ de los participantes, zurdos. Además un 89,7\% ( $\mathrm{n}=1312)$ del total de participantes masculinos eran diestros y en el caso femenino un $92 \%(n=1250)$.

Los análisis chi-cuadrado efectuados (Tabla I) indicaron diferencias estadísticamente significativas entre la variable escoliosis y sexo $\left(\mathrm{X}^{2}=68,267 ; \mathrm{p}<0,001\right)$.

Atendiendo ahora a las variables lateralidad y sexo no se encontraron en este caso diferencias estadísticamente significativas $\left(\mathrm{X}^{2}=4,482 ; \mathrm{p}=0,106\right)$ (Tabla I).

En relación a las variables escoliosis y lateralidad no se hallaron diferencias estadísticamente significativas $\left(\mathrm{X}^{2}=0,792 ; \mathrm{p}=0,673\right)$ (Tabla II). 
FERNÁNDEZ, M.; FERNÁNDEZ, R.; ZURITA, F.; JIMÉNEZ, C.; ALMAGIÀ, A.; YUING, T. \& CURILEM, C. Relación entre escoliosis, sexo y lateralidad manual en una muestra de escolares. Int. J. Morphol., 33(1):24-30, 2015.

Tabla I. Relación entre sexo y escoliosis $\left(X^{2}=68,267 ; \mathrm{p}<0,001\right)$; sexo y lateralidad $\left(\mathrm{X}^{2}=4,482 ; \mathrm{p}=0,106\right)$.

\begin{tabular}{lccccc}
\hline \multicolumn{1}{c}{ Sexo } & \multirow{2}{*}{$\mathbf{n}(\%)$} & \multicolumn{2}{c}{ Escoliosis } & \multicolumn{2}{c}{ Lateralidad } \\
& & Presencia & Ausencia & Diestro & Zurdo \\
\hline Masculino & $1463(51,8)$ & $425(29 \%)$ & $1038(69 \%)$ & $1312(89,7 \%)$ & $151(10,3 \%)$ \\
Femenino & $1359(48,2)$ & $598(44 \%)$ & $761(56 \%)$ & $1250(92 \%)$ & $109(8 \%)$ \\
Total & $2822(100)$ & $1023(36,3 \%)$ & $1799(63,7 \%)$ & $2562(90,8 \%)$ & $260(9,2 \%)$ \\
\hline
\end{tabular}

Tabla II. Relación entre escoliosis y lateralidad manual $\left(\mathrm{X}^{2}=0,792\right.$; $\mathrm{p}=0,673)$.

\begin{tabular}{lccc}
\hline \multirow{2}{*}{ Escoliosis } & \multicolumn{2}{c}{ Lateralidad /n (\%) } & Total / n (\%) \\
& Diestro & Zurdo & \\
\hline Ausencia & $1631(63,7)$ & $168(64,1)$ & $1799(63,7)$ \\
Presencia & $931(36,3)$ & $92(35,9)$ & $1023(36,3)$ \\
Total & $2562(100)$ & $260(100)$ & $2822(100)$ \\
\hline
\end{tabular}

\section{DISCUSIÓN}

En esta investigación se estudiaron 2822 escolares de 6 a 12 años de edad. Esta muestra está en consonancia con otros estudios similares realizados en todo el mundo y que emplearon el cribado como técnica de detección de curvas de tipo escoliótico. En este sentido citamos a Ostojic et al. (2006), que evaluaron 2.517 sujetos en Bosnia-Herzogovina; Zurita Ortega et al. (2008a) analizaron 2.956 escolares en Granada (España) y do Espírito Santo et al. (2011) en Cuiabá (Brasil) valoraron a 3.105 escolares.

La prevalencia de escoliosis encontrada en los participantes fue del $36,3 \%$, lo que sitúa a Almería y su provincia en valores similares a otras zonas españolas y europeas. Así por ejemplo, Redondo Granado et al. (1999) encontraron el $31,6 \%$ y Ostojic et al., observaron un 33,4\%. Por el contrario nuestros datos difieren de lo publicado por Zurita Ortega et al. (2008a) con el 16\%, y Fernández Sánchez et al. (2010), con el 9,4\% en el área metropolitana de Granada. En ocasiones, existen diferencias entre los autores respecto al número de participantes con escoliosis; a este respecto las diferencias pueden estar my relacionadas con el método de medición utilizado, siendo el más objetivo la radiografía (Zurita Ortega et al. (2008a).

En lo que respecta a la distribución por sexos, la prevalencia de escoliosis en niñas fue de un $44 \%$ frente a un $29 \%$ en niños. Estas cifras, por otro lado concuerdan con otros autores como Franco et al. (2007).

En la población estudiada se obtuvo una dominancia manual diestra del 90,8\% y zurda del 9,2\%. Estos resultados están en concordancia con las investigaciones en el ámbito de la lateralidad realizadas por Zurita Ortega et al. (2010).
Respecto al sexo también se encontró un ligero porcentaje a favor de niñas diestras (92\%) respecto a los niños $(89,7 \%)$. De todas formas debemos aclarar que en nuestro caso, los resultados también son debidos a que ciertamente había menos número de niñas zurdas $(8 \%)$ que niños $(10,3 \%)$ y por tanto esta situación pudo influir muy posiblemente en los datos registrados.

En cuanto a la relación entre escoliosis y sexo, se hallaron diferencias estadísticamente significativas $(\mathrm{p}<0,001)$. Así, las niñas presentaron más prevalencia de escoliosis que los niños, a pesar que el número de participantes del sexo masculino era algo mayor (concretamente 104 niños más). Resultados anteriores ya han sido confirmados por otros autores como Franco et al. Desde nuestro punto de vista entendemos que estos resultados pueden ser debidos a que las niñas tienen una mayor flexibilidad (Delgado Valdivia et al.), favorecido entre otros aspectos por un menor desarrollo muscular. Este último factor, la falta de suficiente tono puede motivar una insuficiente fijación de los elementos vertebrales y por tanto predisposición a la escoliosis.

No se encontraron diferencias estadísticamente significativa entre escoliosis y lateralidad manual ( $p=0,673$ ), estando en concordancia con el estudio realizado por Nissinen et al. (1993). Por otro lado, Milenkovic et al. (2004) encontraron que la dominancia manual zurda se asoció significativamente con escoliosis en niñas. Por tanto atendiendo en este caso a nuestra muestra, los resultados parecen indicar que la lateralidad no predispone a la escoliosis y son otros factores los que han podido influir; tal vez los malos hábitos posturales (Colvin et al., 2013), aspectos nutricionales o factores de tipo emocional (Dhalke, 2002; Souchard \& Ollier, 2002).

Atendiendo ahora a las variables lateralidad y sexo no se encontraron en este caso diferencias estadísticamente significativas $(\mathrm{p}=0,106)$. Algunos autores parecen indicar que el sexo no es una variable predisponente en la lateralidad ( $\mathrm{Zu}$ rita Ortega et al., 2010). Otros sin embargo consideran que la zurdera es más frecuente en varones, tal y como sucedió en nuestro estudio. En este sentido barajamos la posibilidad que los padres en ocasiones puedan favorecer la tendencia natural de los niños zurdos, ya que esta situación les puede reportar ventajas en la práctica de deporte mayoritariamente mas- 
culino como el fútbol, boxeo, etc. (Raymond et al., 1996).

Respecto a las limitaciones presente en este estudio debemos indicar que hubiera sido interesante contar con un mayor número de participantes de ambos sexos. Por otro lado también se podían haber utilizado otros instrumentos de medida para evitar en la medida de lo posible falsos positivos. Finalmente indicamos otra última limitación, es decir, no es un estudio longitudinal, por tanto no se han realizado sucesivas mediciones de las variables pertinentes a lo largo de tiempo, afectando muy probablemente a los resultados encontrados en esta ocasión.

\section{CONCLUSIONES}

1) La población escolar de Almería presentó más de un tercio de escoliosis, cifra por otro lado altamente significativa.

2) Se obtuvo un mayor número de escoliosis en niñas debido probablemente a su mayor flexibilidad.
3) La variable lateralidad en los participantes seleccionados no influye en el desarrollo de escoliosis ni está relacionada con el sexo.

4) Atendiendo al número de niños con escoliosis se resalta la importancia en el desarrollo de adecuados programas de intervención para prevenir y tratar esta patología.

\section{AGRADECIMIENTOS}

En primer lugar a cada una de las personas participantes de la investigación, a las Instituciones de Educación participantes por la autorización y facilidades para la realización de la misma y a cada uno de los grupos de trabajo de España y Chile, por la constancia y colaboración en el trabajo. Por otro lado, agradecer a la Dirección de Investigación, Vice-rectoría de Investigación y Estudios Avanzados e Instituto de Biología de la Facultad de Ciencias. Pontificia Universidad Católica de Valparaíso. Chile, por su constante apoyo en materia investigativa.

FERNÁNDEZ, M.; FERNÁNDEZ, R.; ZURITA, F.; JIMÉNEZ, C.; ALMAGIÀ, A.; YUING, T. \& CURILEM, C. Relationship between scoliosis, sex and handedness in a sample of schoolchildren. Int. J. Morphol., 33(1):24-30, 2015.

SUMMARY: Based on the number of problems that develop during childhood, it is clear that scoliosis has an important place because of its impact and in early adulthood. The main objective of the study was to evaluate the relationship between sex, scoliosis and handedness. The sample consisted of 2822 participants, aged between 8 and 12 years, from the province of Almería. Two instruments were used to measure, Inventary Laterality of Edinburgh and Test de Adams, to analyze the selected variables. No statistically significant differences were found between the variables laterality and $\operatorname{sex}(\mathrm{p}=0.106)$ or between laterality and scoliosis $(\mathrm{p}=0.673)$; significant differences however, were found between scoliosis and sex $(\mathrm{p}<0.001)$. Principal conclusions are the importance in the development of appropriate intervention programs bearing in mind the number of children with scoliosis, especially in girls.

KEY WORDS: Scoliosis; Pain; School; Spine; Screening; Prevention.

\section{REFERENCIAS BIBLIOGRÁFICAS}

Álvarez García de Quesada, L. I. \& Nuñez Giralda, A. Escoliosis idiopática. Rev. Pediatr. Aten. Primaria, 13(49):135-46, 2011.

Bull, J. \& Grogan, S. Children having spinal surgery to correct scoliosis: a qualitative study of parents' experiences. J. Health Psychol., 15(2):299-309, 2010.

Bunge, E. M.; Juttmann, R. E.; van Biezen, F. C.; Creemers, H.; Hazebroek-Kampschreur, A. A. J. M.; Luttmer, B. C. F.; Wiegersma, P. A. \& de Koning, H. J. Estimación de la eficacia del cribado de la escoliosis: estudio de casos-controles. Pediatr., 65(1):5-10, 2008.
Castañer, M. \& Camerino, O. La educación física en la enseñanza primaria. Barcelona, Inde, 1993.

Colvin, J. R.; Flores, A. A. A. \& Ilardi, J. S. Anthropometric study of infants in the Chilean public education system for furniture design. Int. J. Morphol., 31(1):189-196, 2013.

Dhalke, R. La enfermedad como símbolo. Barcelona, Robin Book, 2002.

Delgado Valdivia, O.; Martín Cañada, M. A.; Zurita Ortega, F.; Antequera Rodríguez, J. J. \& Fernández Sánchez, M. 
FERNÁNDEZ, M.; FERNÁNDEZ, R.; ZURITA, F.; JIMÉNEZ, C.; ALMAGIÀ, A.; YUING, T. \& CURILEM, C. Relación entre escoliosis, sexo y lateralidad manual en una muestra de escolares. Int. J. Morphol., 33(1):24-30, 2015.

Evolutividad de la capacidad flexora según el sexo y el nivel de enseñanza. Apunts Med. Esport, 44(161):10-7, 2009.

Del Valle Díaz, S. \& De la Vega Marcos, R. Lateralidad en el deporte de full contact. Cambios en diferentes condiciones. Rev. Int. Med. Cienc. Act. Fis. Deporte, 7(25):3251, 2007.

do Espírito Santo, A.; Guimarães, L. V. \& Galera, M. F. Prevalence of idiopathic scoliosis and associated variables in schoolchildren of elementary public schools in Cuiabá, state of Mato Grosso, 2002. Rev. Bras. Epidemiol., 14(2):347-56, 2011.

Fernández Sánchez, M.; Zurita Ortega, F.; Fernández Sánchez, C.; Fernández García, R.; Muñoz-Cruzado y Barba, M. \& Labajos Manzanares, M. T. Prevalencia de escoliosis, dominancia manual lateral y transporte de material en una población masculina de 6-12 años. Apunts. Med. Esport, 45(168):243-9, 2010.

Ferre, E.; Casaprima, V.; Catalán, J. \& Mombiela, J. Técnicas de tratamiento de los trastornos de la lateralidad. Madrid, Lebón, 2006.

Fonseca-Pedrero, E.; Muñiz, J.; Lemos, S.; García-Cueto, E. \& Campillo-Álvarez, A. Lateralidad manual, problemas emocionales y esquizotipia en adolescentes. Psicothema, 19(3):467-72, 2007.

Franco, C. Y.; Guerra, Z. M. \& Otero, M. P. Estudio de caso: terapia manual en una paciente de 18 años con escoliosis juvenil idiopática. Rev. Cienc. Salud, 5(3):78-90, 2007.

Gorman, K. F.; Julien, C. \& Moreau, A. The genetic epidemiology of idiopathic scoliosis. Eur. Spine J., 21(10):1905-19, 2012.

Grauers, A.; Rahman, I. \& Gerdhem, P. Heritability of scoliosis. Eur. Spine J., 21(6):1069-74, 2012.

Healey, J. M. El niño zurdo. Barcelona, Ediciones Medici, 2006.

Jalanko, T.; Rintala, R.; Puisto, V. \& Helenius, I. Hemivertebra resection for congenital scoliosis in young children: comparison of clinical, radiographic, and health-related quality of life outcomes between the anteroposterior and posterolateral approaches. Spine (Phila Pa 1976), 36(1):41-9, 2011.

Kapandji, A. I. Fisiología articular. Tomo 3. Tronco y Raquis.
Madrid, Médica Panamericana, 2007.

Milenkovic, S. M.; Kocijancic, R. I. \& Belojevic, G. A. Left handedness and spine deformities in early adolescence. Eur. J. Epidemiol., 19(10):969-72, 2004.

Miller, J. A.; Nachemson, A. L. \& Schultz, A. B. Effectiveness of braces in mild idiopathic scoliosis. Spine (Phila Pa 1976), 9(6):632-5, 1984.

Monge Crespo, M. C. El alumno zurdo: didáctica de la escritura. Zaragoza, Mira Editores, 2000.

Mcmanus, C. Mano derecha, mano izquierda: los orígenes de la asimetría en cerebros, cuerpos, átomos y culturas. Barcelona, Intervención Cultural, 2007.

Nissinen, M.; Heliövaara, M.; Seitsamo, J. \& Poussa, M. Trunk asymmetry, posture, growth, and risk of scoliosis. A three-year follow-up of Finnish prepubertal school children. Spine (Phila Pa 1976), 18(1):8-13, 1993.

Oldfield, R. C. The assessment and analysis of handedness: the Edinburgh inventory. Neuropsychologia, 9(1):97113, 1971.

Ostojic, Z.; Kristo, T.; Ostojic, L.; Petrovic, P.; Vasilj, I.; Santic, Z.; Maslov, B.; Vasilj, O. \& Caric, D. Prevalence of scoliosis in school-children from Mostar, Bosnia and Herzegovina. Coll. Antropol., 30(1):59-64, 2006.

Pärtan, G.; Eyb, R. \& Artacker, G. Imaging of non-traumatic spinal diseases in children. Radiologe, 50(12):1107-14, 2010.

Pérez-Caballero Macarrón, C.; Burgos Flores, J.; Martos Sánchez, I.; Pérez Palomino, A.; Vázquez Martínez, J. L.; Álvarez Rojas, E.; Fernández Pineda, L. \& Vellibre Vargas, D. Early postoperative complications after scoliosis surgery. An. Pediatr. (Barc.), 64(3):248-51, 2006.

Raymond, M.; Pontier, D.; Dufour, A. B. \& Moller, A. P. Frequency-dependent maintenance of left handedness in humans. Proc. Biol. Sci., 263(1377):1627-33, 1996.

Redondo Granado, M. J.; Arnillas Gómez, P. \& Fernández Alonso, C. Screening for adolescent idiopathic scoliosis: is current knowledge sufficient to support its use? An. Esp. Pediatr., 50(2):129-33, 1999.

Rigal, R. Motricidad humana. Fundamentos y aplicaciones pedagógicas. Madrid, Pila Teleña, 1988. 
Sato, T.; Hirano, T.; Ito, T.; Morita, O.; Kikuchi, R.; Endo, N. \& Tabane, N. Back pain in adolescents with idiopathic scoliosis: epidemiological study for 43,630 pupils in Niigata City, Japan. Eur. Spine J., 20(2):274-9, 2011.

Souchard, P. \& Ollier, M. Escoliosis, su tratamiento en fisioterapia y ortopedia. Madrid, Médica Panamericana, 2002.

Springer, S. P. \& Deutsch, G. Cerebro izquierdo, cerebro derecho. Barcelona, Gedisa, 2006.

Terrones González, A.; Salvador Moysén, J. \& Estrada Martínez, S. Prevalencia de la dominancia lateral en escolares de la ciudad de Durango. Salud Durango, 4(2):510, 2003.

Tichy, J. \& Belacek, J. Laterality in children: cerebellar dominance, handedness, footedness and hair whorl. Act. Nerv. Super. Rediviva, 51(1-2):9-20, 2009.

Wang, W.; Zhu, Z.; Zhu, F.; Sun, C.; Wang, Z.; Sun, X. \& Qui, Y. Different curve pattern and other radiographical characteristics in male and female patients with adolescent idiopathic scoliosis. Spine (Phila Pa 1976), 37(18):1586-92, 2012.

Watanuki, A.; Yamada, H.; Tsutsui, S.; En-yo, Y.; Yoshida, M. \& Yoshimura, N. Radiographic features and risk of curve progression of de-novo degenerative lumbar scoliosis in the elderly: a 15-year follow-up study in a community-based cohort. J. Orthop. Sci., 17(5):526-31, 2012.

Yagi, M.; King, A. B. \& Boachie-Adjei, O. Incidence, risk factors, and natural course of proximal junctional kyphosis: surgical outcomes review of adult idiopathic scoliosis. Minimum 5 years of follow-up. Spine (Phila Pa 1976), 37(17):1479-89, 2012.

Zurita Ortega, F.; Moreno Lorenzo, C.; Ruiz Rodríguez, L.; Martínez Martínez, A.; Zurita Ortega, A. \& Castro Sánchez, A. M. Screening of scoliosis in a school population of 8 to 12 years in the province of Granada (Spain). An. Pediatr. (Barc.), 69(4):342-50, 2008a.

Zurita Ortega, F.; Romero Cerezo, C.; Ruiz Rodríguez, L.; Martínez Martínez, A.; Fernández García, R. \& Fernández Sanchez, M. Influencia de las alteraciones raquídeas en la flexibilidad de los escolares. Rev. Int. Med. Cienc. Act. Fís. Deporte, 8(32):282-98, 2008b.

Zurita Ortega, F.; Fernández García, R.; Rojas Ruiz, F. J. \&
Cepero González, M. Lateralidad manual y variables geográficas, antropométricas, funcionales y raquídeas. Rev. Int. Med. Cienc. Act. Fis. Deporte, 10(39):439-57, 2010.

Dirección para Correspondencia:

Klgo. MSc. Tuillang Yuing Farías

Laboratorio de Antropología Física y Anatomía Humana

Instituto de Biología, Facultad de Ciencias

Pontificia Universidad Católica de Valparaíso

Valparaíso

CHILE

Email: yuenglam@gmail.com

Recibido : 06-08-2014

Aceptado: 05-11-2014 